\title{
Effects of High Beam Rates on TPC's*
}

A. Etkin, S.E. Eiseman, K.J. Foley, R.W. Hackenburg, R.S. Longacre W. A. Love, T.W. Morris, E.D. Platner and A.C. Saulys

Brookhaven National Laboratory, Upton, New York 11973

\section{S.J. Lindenbaum}

Brookhaven National Laboratory and City College of New York

T.J. Hallman

University of California, Los Angeles, California 90024

C.S. Chan, M.A. Kramer, K.H. Zhao and Y. Zhu

City College of New York, New York, New York 10031

L. Madansky

Johns Hopkins University, Baltimore, Maryland 21218

G. Rai

Lawrence Berkeley Laboratory, Berkeley, California 94720

S. Ahmad, B.E. Bonner, J.A. Buchanan, C.N. Chiou, J.M Clement

G.S. Mutchler, and J.B. Roberts

Bonner Nuclear Laboratory, Rice University, Houston, Texas 77251

Proc. of the $6^{\text {th }}$ Wire Chamber Conference

Vienna, Austria

February 17-21, 1992

February 6, 1992

* This research was supported by the U.S. Department of Energy under Contract Nos. DEAC02-76CH00016, DE-AC02-83ER40107, DE-FG03-88ER40424, DE-SG02-88ER40413, DE-AC03-76SF00098, DE-FG05-87ER40309, and the City University of New York PSCBHE Research Award Program. 


\title{
Effects of High Beam Rates on TPC's*
}

A. Etkin, S.E. Eiseman, K.J. Foley, R.W. Hackenburg, R.S. Longacre

W. A. Love, T.W. Morris, E.D. Platner and A.C. Saulys

Brookhaven National Laboratory, Upton, New York 11979

\section{S.J. Lindenbaum}

Brookhaven National Laboratory and City College of New York

T.J. Hallman

University of California, Los Angeles, California 90024

C.S. Chan, M.A. Kramer, K.H. Zhao and Y. Zhu

City College of New York, New York, New York 10031

L. Madansky

Johns Hopkins University, Baltimore, Maryland 21218

G. Rai

Lawrence Berkeley Laboratory, Berkeley, California 94720

S. Ahmad, B.E. Bonner, J.A. Buchanan, C.N. Chiou, J.M Clement G.S. Mutchler, and J.B. Roberts

Bonner Nuclear Laboratory, Rice University, Houston, Texas 77251

\begin{abstract}
The TPC's (Time Projection Chamber) used in E-810 at the AGS (Alternating Gradient Synchrotron) were exposed to silicon ion fluxes equivalent to more than $10^{7}$ minimum ionizing particles per second to measure the distortion of the electric field caused by positive ions in the drift region. Results of these tests are presented and the consequences for the TPC based experiment at RHIC (Relativistic Heavy Ion Collider) are discussed.
\end{abstract}

* This research was supported by the U.S. Department of Energy under Contract Nos. DEAC02-76CH00016, DE-AC02-83ER40107, DE-FG03-88ER40424, DE-SG02-88ER410413, DE-AC03-76SF00098, DE-FG05-87ER40309, and the City University of New York PSCBHE Research Award Program. 


\section{Introduction}

In order to study the performance of TPC's in a high particle flux environment we have made a number of measurements utilizing the AGS experiment E-810 TPC's [1]. This experiment uses 3 TPC modules in a vertical dipole magnetic field (Fig, 1). Silicon and proton beams can be directed to a variety of nuclear targets ranging from $S i$ to $P b$. In the forward hemisphere the experiment has high acceptance and has reconstructed events with up to 130 charged particle tracks from a heavy target. Measurements of the shift in measured track positions due to ion loading will be given. Application of these results to the question of luminosity limitation capability of the "STAR" [2] experiment at RHIC will be discussed.

\section{Distortions Due To Ion Loading}

Figure 2 shows a head on view of an E-810 TPC. The drift field is $\approx 300 \mathrm{volts} / \mathrm{cm}$ and the gas is $60 \%$ argon, $15 \%$ isobutane and $5 \%$ dimethoxymethane. The ion beam passes directly through the center of the TPC. Typically the beam is $1.5 \mathrm{~cm}$ wide by $0.6 \mathrm{~cm}$ high in the center of the first TPC module. The AGS beam has a spill duration of 1 second. Some time after the beam starts positive ions created at the beginning of the spill reach the negative HV electrode. At this point a sheet of ions has formed from the beam to the HV electrode. This produces a distortion of the "uniform" electric field. In fact the electrons that are drifting downward will see a reduction in electric field near the beam causing the drift velocity to be reduced in the vicinity of the beam. We have studied this effect at three different beam rates $67 \mathrm{~K}$ (high), $40 \mathrm{~K}$ (medium) and $14 \mathrm{~K}$ (low) $S i$ ions per one second beam spill. At the highest rate with the small beam $(1.5 \mathrm{~cm}$ by $0.6 \mathrm{~cm})$ a positive ion sheet with a density of the order of $10^{7} \mathrm{ion} / \mathrm{cm}^{3}$ is generated along the beam as shown in Fig. 2 . Two distortions were measured, the first is in the drift direction $Y$ and the second is in the horizontal direction $X$. In order to measure these effects the difference between the projected beam track position and the position determined in the 'TPC' is calculated. Projected heam track positıons are determined using two sets of $Y$ and $Y$ measuring beam PWC's. A run at low rate $(4 \mathrm{~K}$ ions per spill) with a defocussed beam $(10 \mathrm{~cm}$ wide by $12 \mathrm{~cm} \mathrm{high})$ is used 
to determine the relationship between row numbers, wire numbers and drift time, and the trajectory position in the TPC. The differences $\Delta X$ and $\Delta Y$ (projected position - position measured in TPC) are calculated for subsequent runs. Figure 3 shows $\Delta Y$ in a high rate run at the center of the first TPC module (row \#6) as a function of event number during the spill with $30 \mathrm{~ms}$ between events. As can be seen, after $\sim 300 \mathrm{~ms}$ the distortion reaches a maximum and remains relatively constant until near the end of the spill when the beam intensity drops. This is consistent with the development of an ion sheet during this time that reaches equilibrium after $\sim 300 \mathrm{~ms}[3]$.

Each TPC has 12 anode rows surrounded by a field cage. In Fig. 4 we plot the difference, $\Delta Y$, versus row number in each module [4] measured using only events that occur after $360 \mathrm{~ms}$ into the spill for a small high rate beam. One sees that the first and last rows (nearest to the field cage) have smaller differences than in the middle. This appears to be because of the proximity of the field cage which reduces the electric field distortion caused by the ion sheet. Figure 5 shows $\Delta Y$ versus row number measured in a magnetic field of $0.5 T$ with a small high rate beam and is very similar to the field free case. In both cases $\Delta Y$ is negative indicating a reduction of the effective drift velocity as one would expect because the electrons are attracted by the positive ion sheet. Similar results were found for the medium and low rate small beam zero magnetic field data. In order to compare the effect at different beam rates we have used the average of the difference between the shift at the edges and that at the middle [5] (bow) instead of the absolute value of $\Delta Y$ which is much more sensitive to changes in the a.verage drift velocity caused by environmental changes. Figure 6 shows the bow versus beam rate. We have been able to calculate the shape of the effect of the positive ions with the same electrostatics program used in the development of the "STAR" proposal [2] and have good agreement as shown in Figs. 4, 5 and 6 [6]. Figure 7 shows the $\Delta . I$ measured in the middle of the first TPC module, versus beam $X$ for the small high rate beam and zero magnetic field. $\Delta X$ is largest near the edge of the beam and zero at the beam center and is positive for $X<0$ and negative for $X>0$ as expected if the drifting electrons are attracted to the positive ion sheet. 
At RHIC the "STAR" central TPC will have a minimum ionizing track rate of $2 \times 10^{6}$ tracks per second for $A u-A u$ collisions. At the inner radius this is spread out over an effective area for positive ion buildup of $7.5 \mathrm{~m}^{2}$ or an average of 12 tracks per $\mathrm{cm}^{2}$ per second and a maximum positive ion density of $\approx 700 \mathrm{ion} / \mathrm{cm}^{3}$. The $\mathrm{E}-810$ test had a track density of $>10^{7}$ minimum ionizing tracks per second in a $1 \mathrm{~cm}^{2}$ spot and positive ion density of $10^{7} \mathrm{ion} / \mathrm{cm}^{3}$. This ion density caused a distortion in the drift direction of only the order of $1 \mathrm{~mm}$. In addition the ability of the "STAR" electrostatics program to calculate shifts in agreement with our measurements gives ls additional confidence in its prediction that the TPC will be able to operate satisfactorily at significantly higher luminosity than the RHIC design value.

\section{Conclusions}

Distortions due to ion loading in the E-810 TPC's have been found to be small even at very high effective particle rates. This result supports the use of TPC's at RHIC even at the highest planned luminosity.

\section{References \& Footnotes}

(1) A. Etkin, et al. "Modular TPC's for Relativistic Heavy-Ion Experiments", Nuclear Instruments and Methods, Vol. A2837, No. 3, pp. 557-566, November 1989. A. Etkin, et al. "A TPC for Large Solid Angle Relativistic Heavy Ion Experiments", IEEE Transactions on Nuclear Science, Vol. 36, No. 1, Part I, pp. 58, February 1988.

[2] "STAR", Solenoidal Tracker At RHIC, approved major detector for RHIC, see LBL31040, 1991 and LBL-29651, 1990. K. Kadija, G. Paic, D. Vranic - Rudjer Boskovic Institute; G. Danby, S. Eiseman, A. Etı:in, K.J. Foley, R.W. Hackenburg, M.J. Leline, R.S. Longacre, W. A. Love, E.D. Platner, A.C. Saulys, J.H. Van Dijk - Brookhaven National Laboratory; F.P. Brady, J.E. Draper, J.L. Romero - University of California at Davis; J.B. Carroll, V. Cihazikhanian, E. (inlmez, T.J. Hallman, (i.J. Igo, S. Trentalange, C.A. Whitten, Jr. - University of California at Los Angeles; M. Kaplan, P. Karol, Z. Milosevich, E. Vardaci - Carnegie-Melon University; M.G. Cheruey - Creighton 
University; S. Margetis, R.E. Renfordt, D. Röehrich, R. Stock, H. Ströebele, S. Wenig University of Frankfurt; L. Madansky - The Johns Hopkins University; B.D. Anderson, D. Keane, R. Madey, J. Watson - Kent State University; F. Bieser, M.A. Bloomer, D.A. Cebra, W. Christie, E. Friedlander, D. Greinex, C. Gruhn, J.W. Harris, H. Huang, P.M. Jacobs, S.A. Kleinfelder, P.J. Lindstrom, H. Matis, C. McParland, R. Morse, C.J. Naudet, G. Odyniec, D.L. Olson, A.M. Poskanzer, G. Rai, H.-G. Ritter, I. Sakrejda, J.O. Schambach, L.S. Schroeder, P.A. Seidl, T.M. Symons, H. Wieman, W.K. Wilson - Lawrence Berkeley Laboratory; C.S. Chan, M.A. Kramer, S.J. Lindenbaum - City College of New York; A. Aprahamian, N.N. Biswas, U. Garg, V.P. Kenney, J. Piekarz - University of Notre Dame; T. Humanic University of Pittsburgh; D.D. Carmony, Y. Choi, A. Hirsch, E. Hjort, N.T. Porile, R.P. Scharenberg, B. Srivastava, M.L. Tincknell - Purdue University; D.L. Adams, S. Ahmad, B.E. Bonner, J.A. Buchanan, C.N. Chiou, J.M. Clement, M.D. Corcoran, T. Empl, H.E. Miettinen, G.S. Mutchler, J.B. Roberts, J. Skeens, I. Stancu - Rice University; A.D. Chacon, K.L. Wolf - Texas A \& M University; W. Dominik, M. Gazdzicki - Warsaw University; T. Pawlak, W. Peryt, J. Fluta - Warsaw University of Technology; W.J. Braithwaite, J.G. Cramer, D. Prindle, T. Trainor, University of Washington; A. Breskin, R. Chechik, Z. Fraenkel, I. Tserruya - Weizmann Institute of Science.

(3) The time to reach equilibrium is a combination of the observed rise time of the beam spill $(<100 \mathrm{~ms})$ and the drift time for the positive ions from the center of the TPC $(\approx$ $250 \mathrm{~ms})$.

[4] Rows $7 \& 8$ in the second module were not working and are not plotted.

[5] Bow $=\frac{1}{6} \sum_{\text {modules }}\left(\Delta Y_{\text {row } 1}+\Delta Y_{\text {row 12 }}-\Delta Y_{\text {row 6 }}-\Delta Y_{\text {row 7 }}\right)$

[6] A positive ion drift velocity of $138 \mathrm{~cm} / \mathrm{sec}$ was used. This value is consistent with the time development of the shift at the start of spill. The Bow is proportional to the beam rate and inversely proportional to the positive ion drift velocity. 


\section{Figure Captions}

Figure 1: Plan view of E-810 apparatus.

Figure 2: Section of a TPC looking along the beam showing beam spot and positive ion sheet.

Figure 3: $\Delta Y$ versus spill event number for small beam and high rate.

Figure 4: $\Delta Y$ versus module row number for small beam and high rate.

Figure 5: $\Delta Y$ versus row number for small beam, high rate and $0.5 T$ field.

Figure 6: "Bow" versus beam rate.

Figure 7: $\Delta X$ versus projected $X$ for small beam and high rate. 


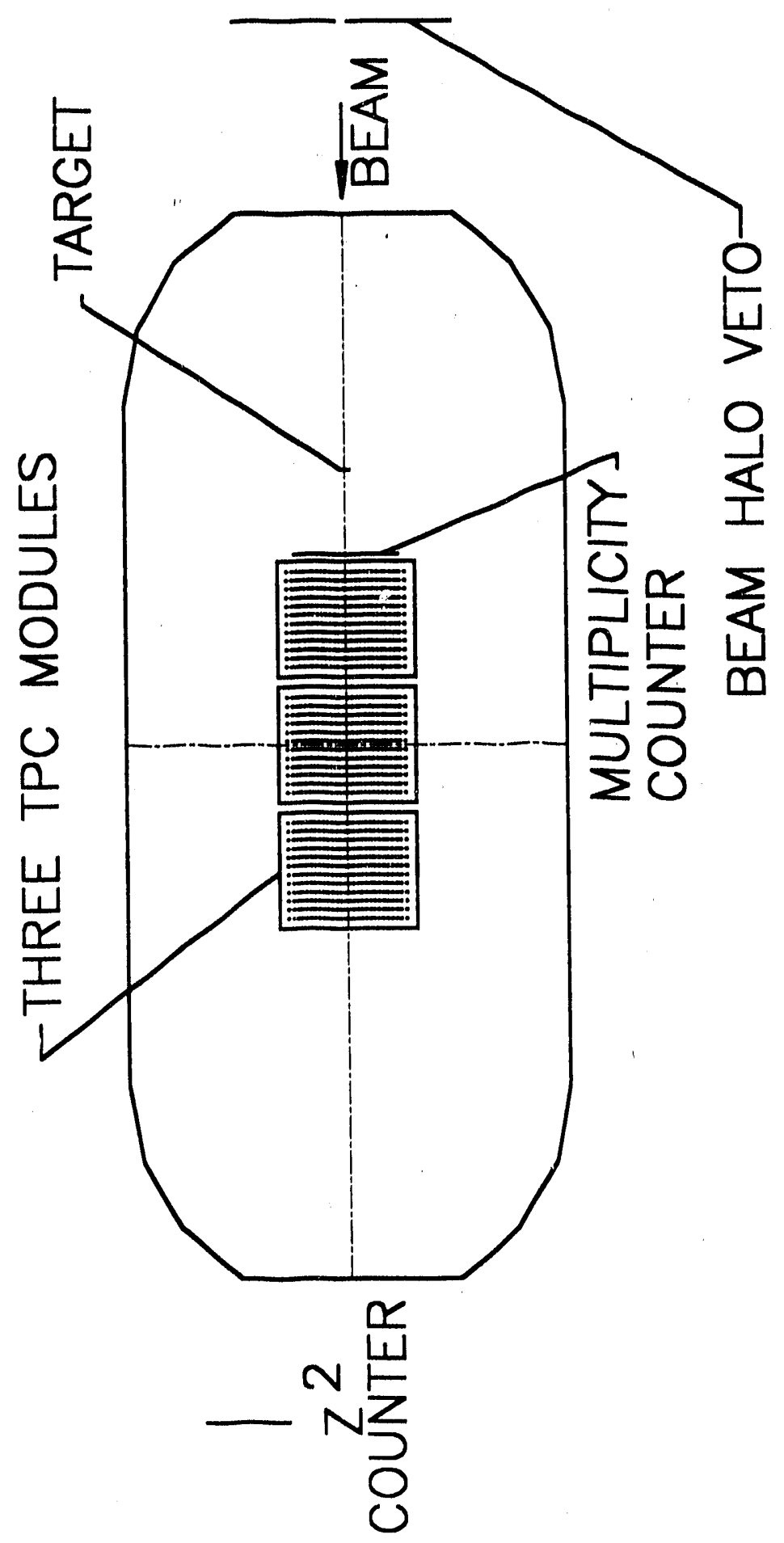

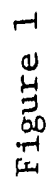




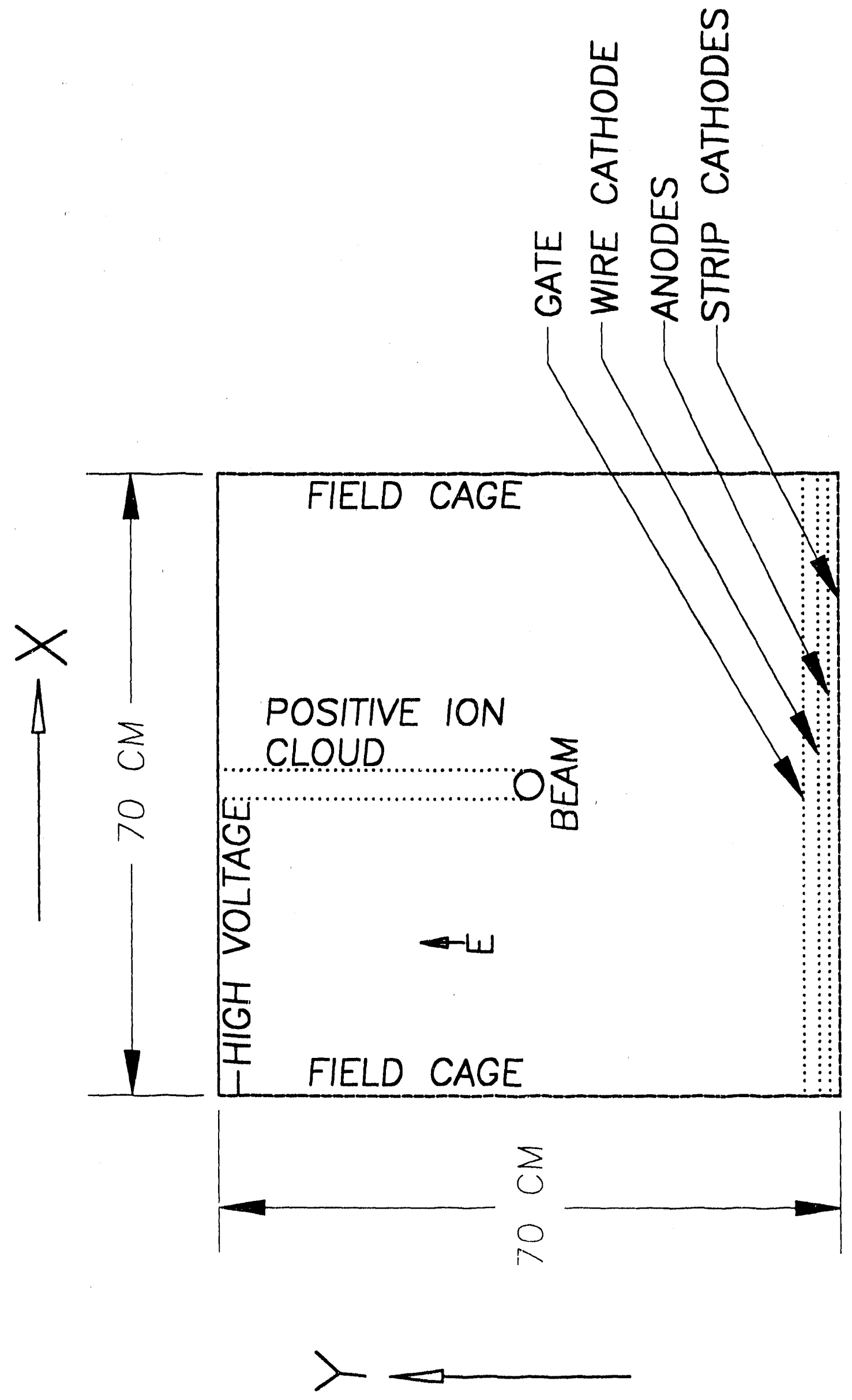




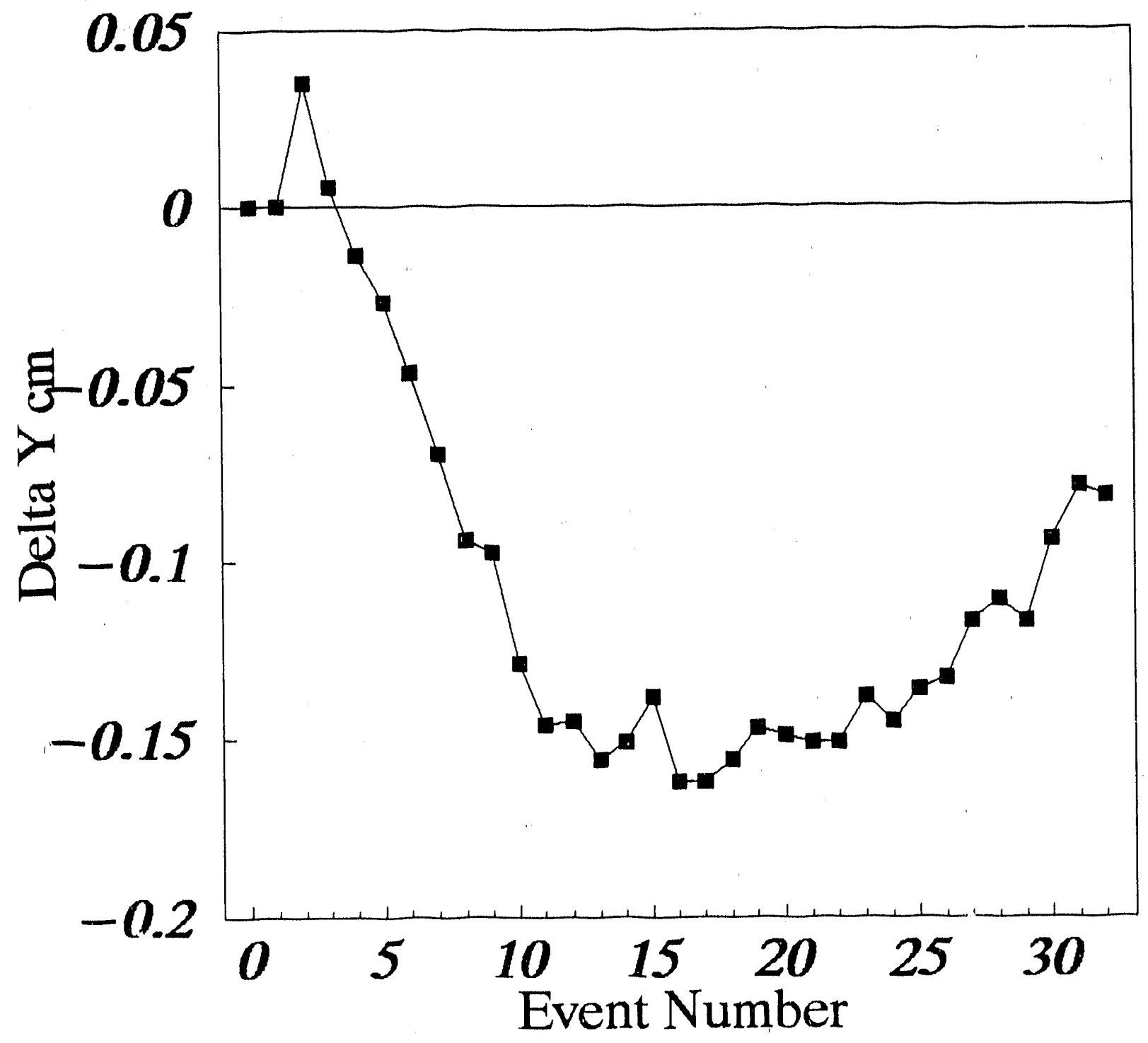

[fig 3] 


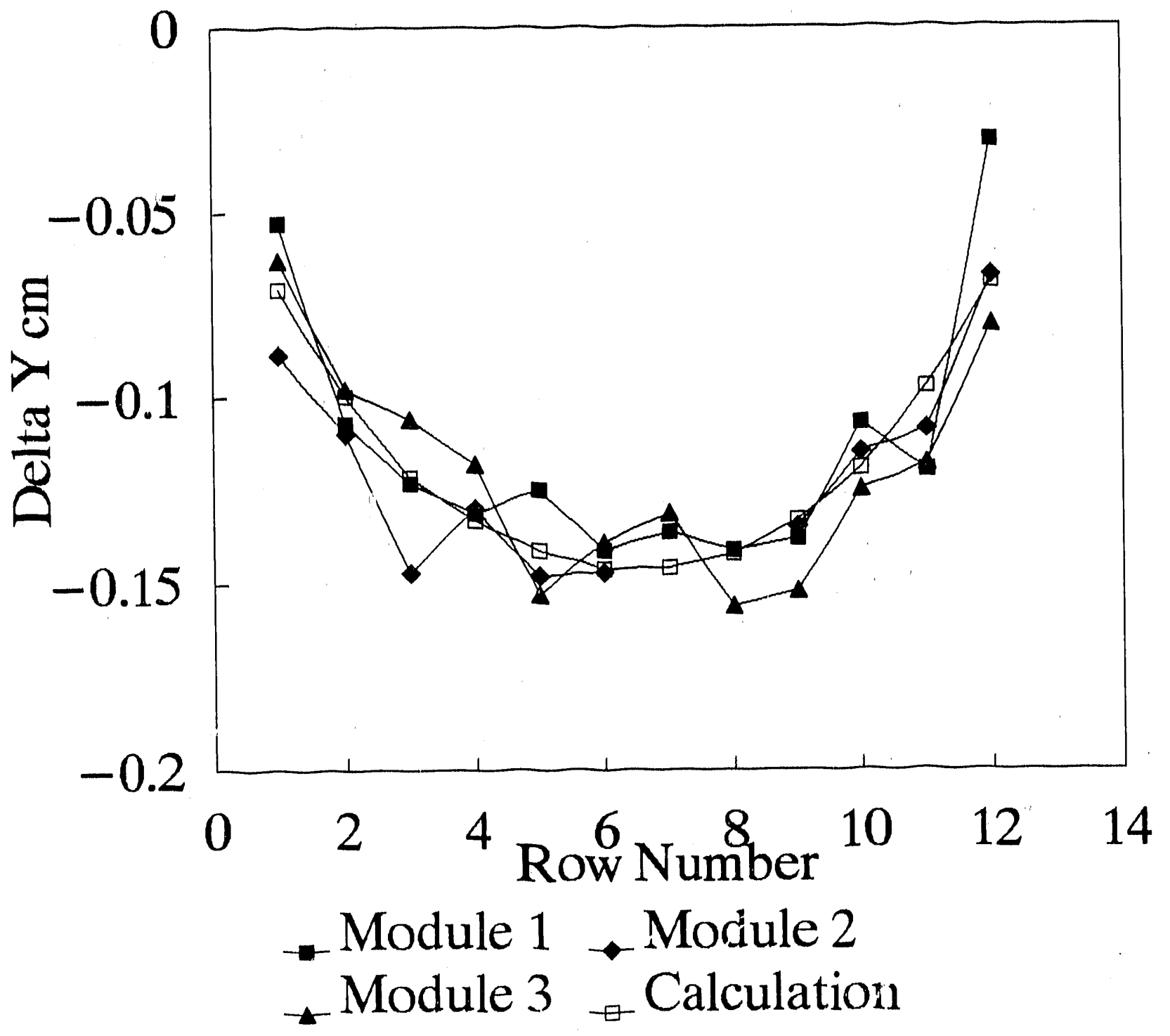

[fig 4] 


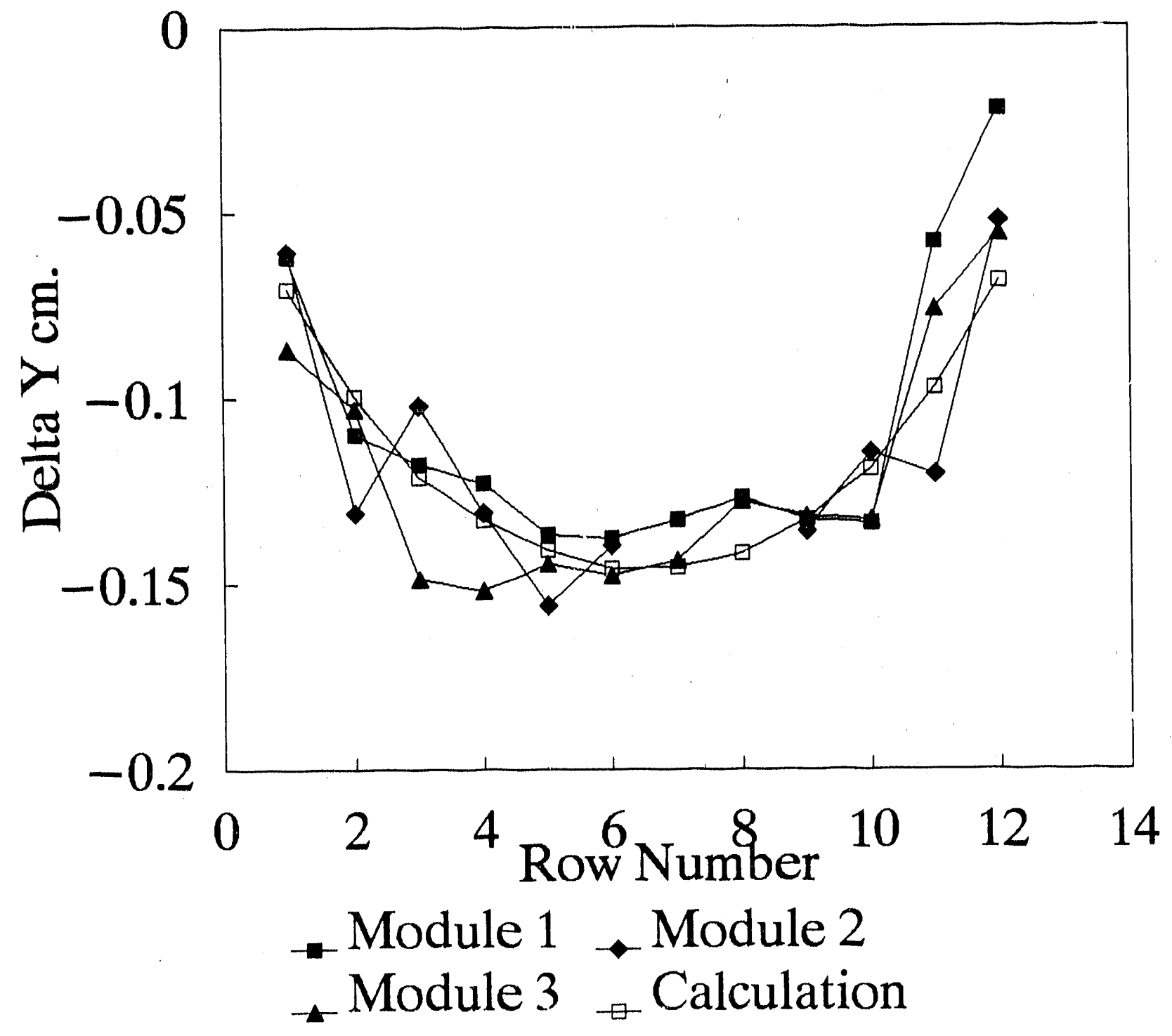

[fig 5] 


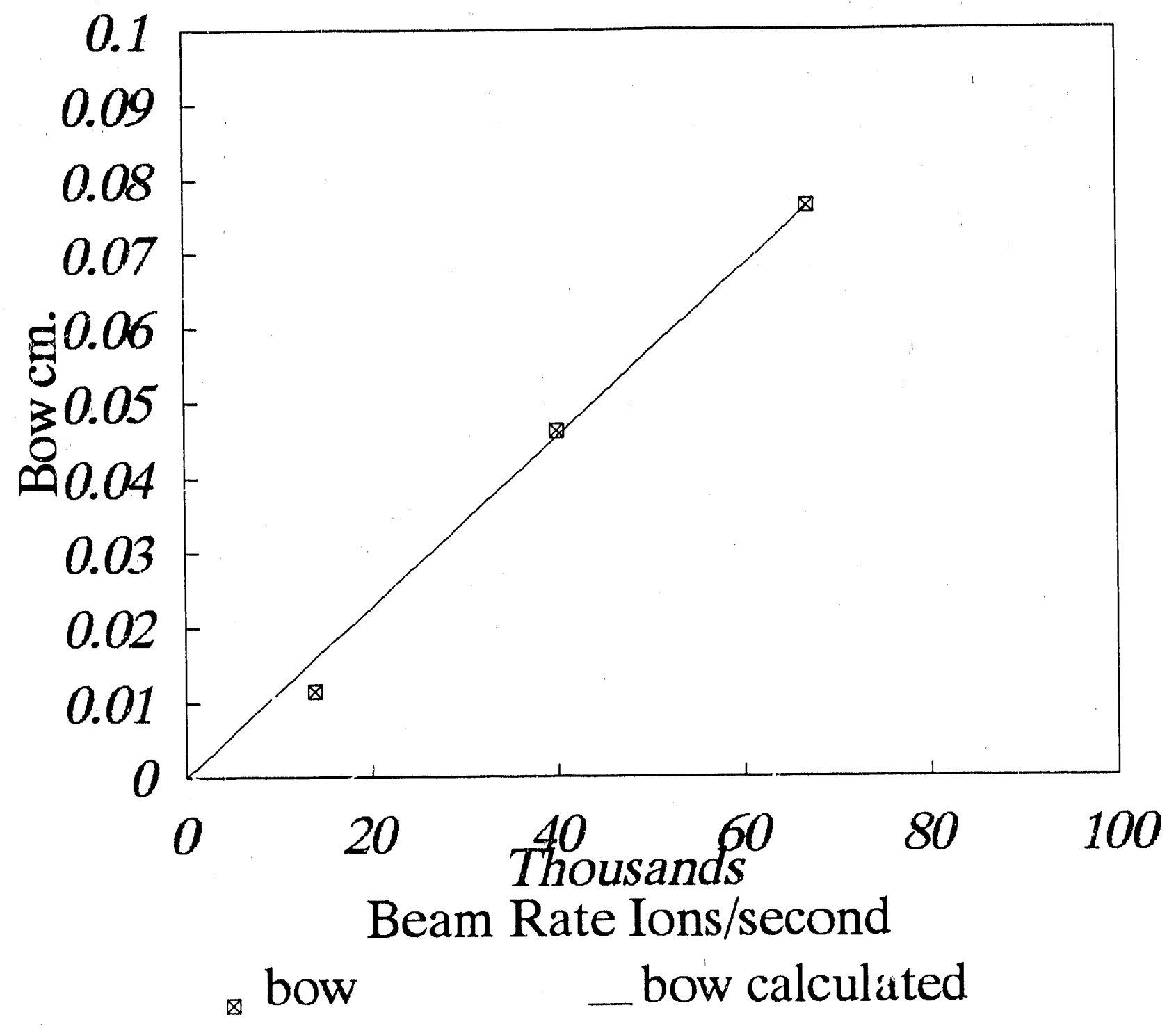

[fig 6] 


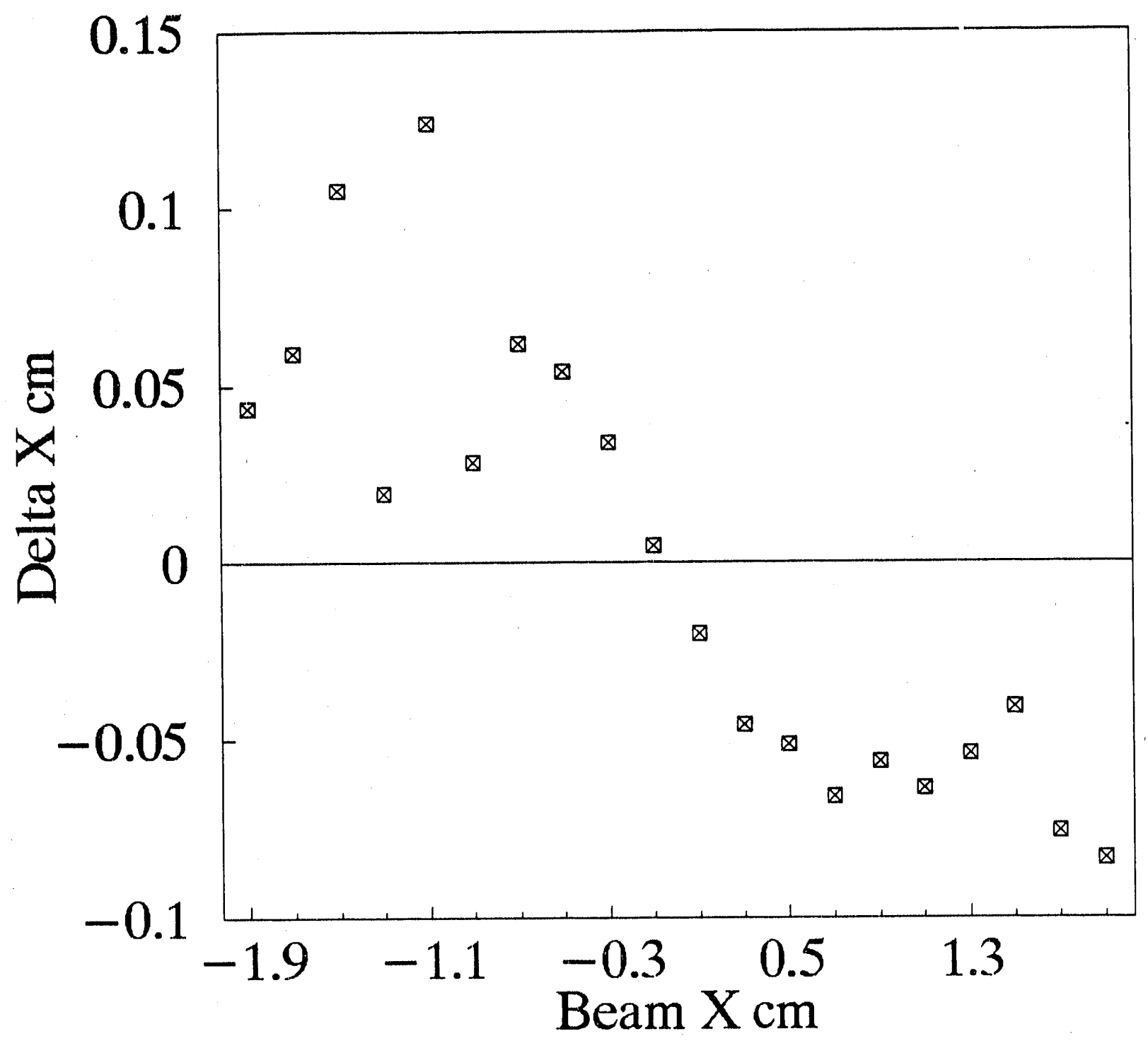

[fig 7] 

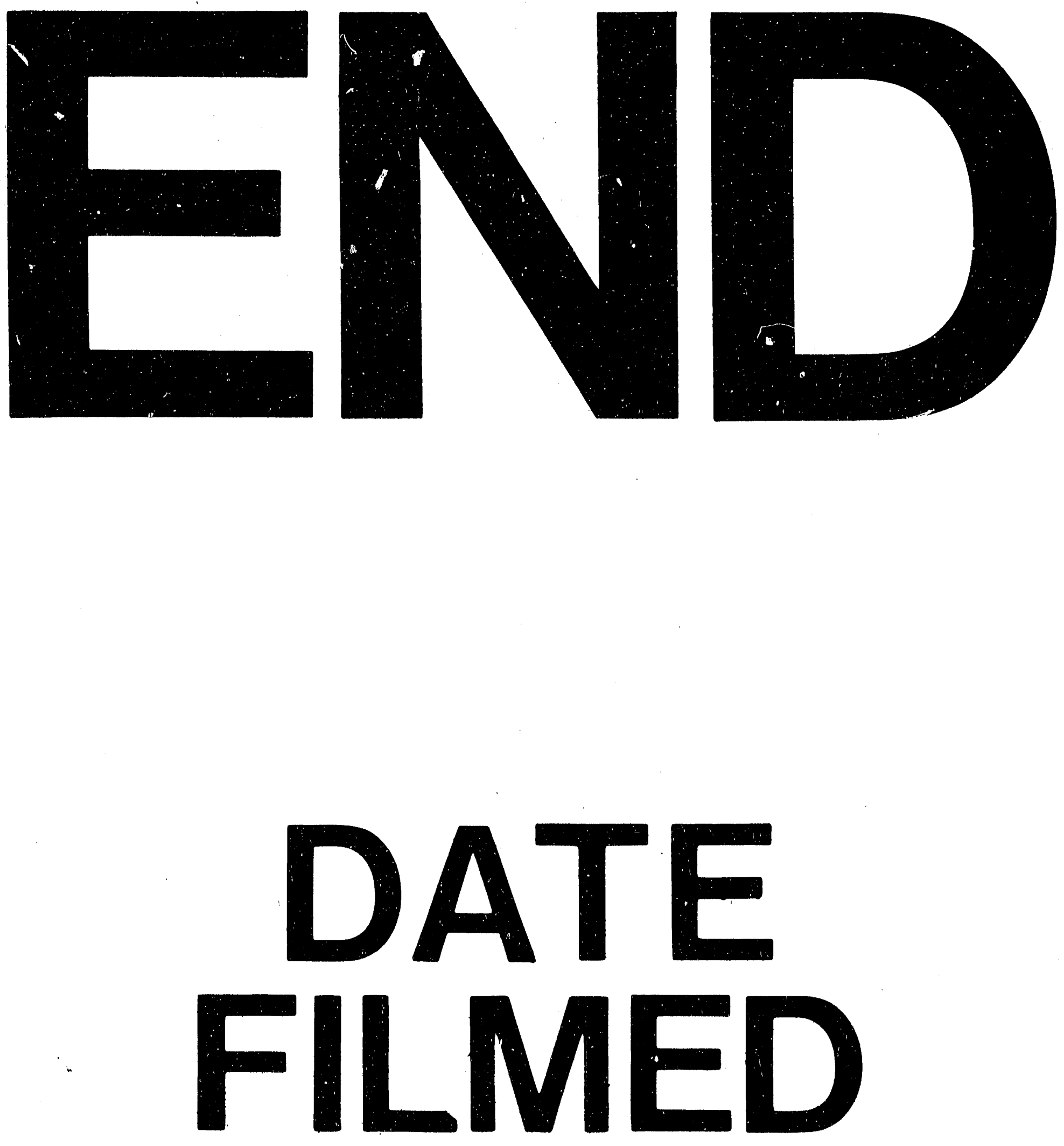

$+$

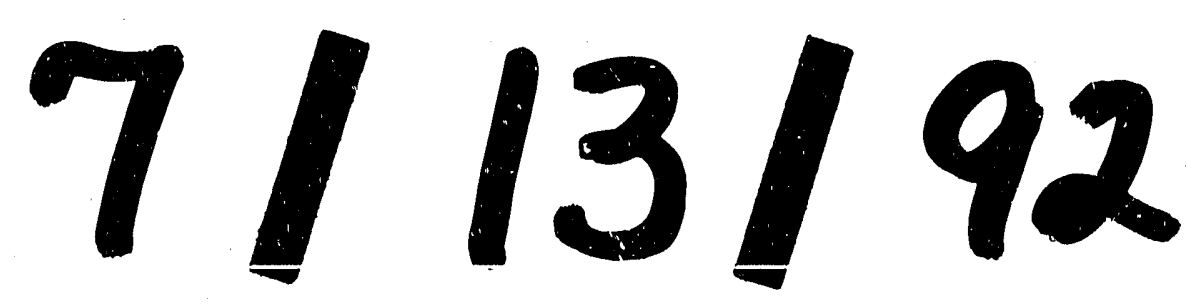




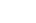

